\title{
Search for dark matter produced in association with a hadronically decaying $Z$ ' vector boson with the ATLAS detector at the LHC
}

\section{Philipp Gadow*, on behalf of the ATLAS collaboration}

Max-Planck-Institut für Physik, Föhringer Ring 6, D-80805 Munich, Germany

E-mail: paul.philipp.gadow@cern.ch

\begin{abstract}
A search for dark matter pair production in association with a $Z^{\prime}$ boson is performed using $36.1 \mathrm{fb}^{-1}$ of LHC $p p$ collision data at $\sqrt{s}=13 \mathrm{TeV}$ recorded with the ATLAS detector. Events are characterised by large missing transverse momentum and a hadronically decaying vector boson reconstructed as either a pair of small-radius jets, or as a single large-radius jet with a twobody decay substructure. Results are interpreted in terms of simplified models which describe the interaction of dark matter and Standard Model particles.
\end{abstract}

Sixth Annual Conference on Large Hadron Collider Physics (LHCP2018)

4-9 June 2018

Bologna, Italy

${ }^{*}$ Speaker. 


\section{Introduction}

A search for dark matter is performed under the yet unexplored hypothesis of the dark matter particle production in association with a hadronically decaying new vector boson $Z^{\prime}$ using $36.1 \mathrm{fb}^{-1}$ of $p p$ collision data collected at a centre-of-mass energy of $\sqrt{s}=13 \mathrm{TeV}$ [1] recorded by the ATLAS detector [2] at the Large Hadron Collider [3]. The results of the search are interpreted in terms of a dark-fermion model and a dark-Higgs model [4] with couplings $g_{\mathrm{SM}}=0.1$ of the mediator to the Standard Model and $g_{\mathrm{DM}}=1.0$ to dark-sector particles. Both a light dark-sector scenario with the dark-fermion masses set to $m_{\chi_{1}}=5 \mathrm{GeV}, m_{\chi_{2}}=m_{Z^{\prime}}+30 \mathrm{GeV}$ and the dark-Higgs mass set to $m_{h_{D}}=\min \left(m_{Z^{\prime}}, 125 \mathrm{GeV}\right)$, and a heavy dark-sector scenario with the masses set to $m_{\chi_{1}}=0.5 m_{Z^{\prime}}$, $m_{\chi_{2}}=2 m_{Z^{\prime}}$ and $m_{h_{D}}=\max \left(125 \mathrm{GeV}, m_{Z^{\prime}}\right)$, respectively, are considered. Figure 1 shows Feynman graphs illustrating the dark-fermion (left) and the dark-Higgs (right) signal models. The dominant background processes are $Z(v \bar{v}, \tau \bar{\tau})+$ jets, $W(\ell v)+$ jets, and $t \bar{t}$ production.
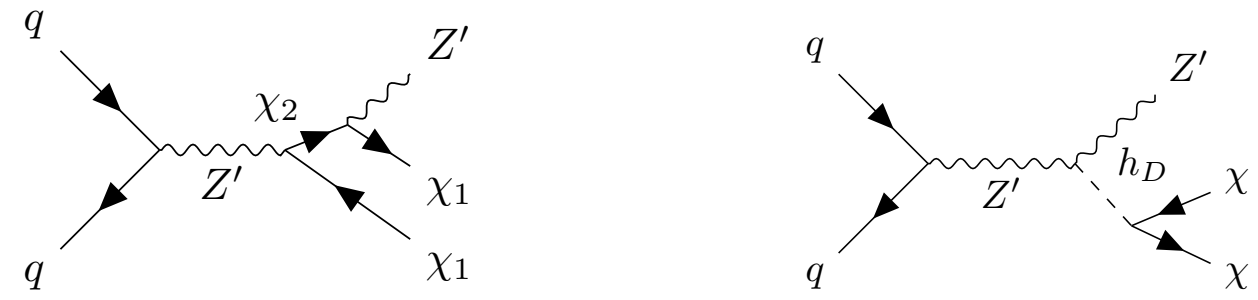

Figure 1: Representative Feynman diagrams for the dark matter particle $(\chi)$ pair-production in association with an additional heavy dark-sector fermion (left) or via a dark-sector Higgs boson $\left(h_{D}\right)$ decay (right).

\section{Event selection}

The final state is characterized by large missing transverse momentum $E_{\mathrm{T}}^{\text {miss }}$ from a pair of dark matter particles and by the jets from the hadronic decay of a hypothetical new $Z^{\prime}$ boson. The $Z^{\prime}$ decay products are reconstructed from calorimeter energy clusters using the anti- $k_{\mathrm{T}}$ jet clustering algorithm [5], either as a pair of small-radius jets with radius parameter $R=0.4$ (resolved topology) or in the case of a highly boosted $Z^{\prime}$ boson for which the small-radius jets start to merge into one, as a single large-radius jet with $R=1.0$ (merged topology). The event selection targeted at the merged (resolved) topology requires $E_{\mathrm{T}}^{\text {miss }}>250 \mathrm{GeV}(150 \mathrm{GeV})$ and a large-radius jet (dijet system) with an invariant mass in the range of the $Z^{\prime}$ boson mass. Since the $Z^{\prime}$ boson mass $m_{Z^{\prime}}$ is a free model parameter the search is performed with a sliding $m_{Z^{\prime}}$ mass window between 80 and $500 \mathrm{GeV}$. The events are selected by the combination of $E_{\mathrm{T}}^{\text {miss }}$ triggers with thresholds between $70 \mathrm{GeV}$ and $110 \mathrm{GeV}$, a veto on events containing electrons or muons, and further requirements on the kinematic properties of the decay products. For masses $m_{Z^{\prime}}>100 \mathrm{GeV}$, only the resolved topology is considered. For lower masses the merged topology selection is applied first and the resolved topology selection only on the remaining events. Events are further classified according to the number of $b$-tagged jets into categories with zero, one, and two $b$-jets to improve the sensitivity of the search. The $b$-tagging within the large-radius jets is based on track-based subjets with radius parameter $R=0.2$. The merged selection additionally takes into account the jet substructure information to distinguish high and low purity signal regions. 


\section{Background estimation}

In addition to these selection criteria defining the signal region, two background-enriched control regions are defined to improve the original simulation-based estimate of the dominant background processes. The control regions are designed to be disjoint to the signal region by requiring the presence of one (two) charged lepton(s) to estimate the $W(\ell v)+$ jets and $t \bar{t}(Z(v \bar{v}, \tau \bar{\tau})+$ jets) process(es). Other background processes such as single-top quark and diboson production are estimated from a detailed simulation of the detector response. Only the multijet background is determined solely from data due to very large mismodelling uncertainties.

\section{Results}

The distribution of $E_{\mathrm{T}}^{\text {miss }}$, the final discriminant for the statistical interpretation of data, is shown in Figure 2 for various signal region categories after the final event selections targeting $Z^{\prime}$ resonances with masses of $90 \mathrm{GeV}$ and $350 \mathrm{GeV}$. No significant deviation from the Standard Model background prediction is observed. The largest excess of the data above the expectation, corresponding to a local (global) significance of $3 \sigma(2.2 \sigma)$, is observed for a hypothesized signal at $m_{Z^{\prime}}=350 \mathrm{GeV}$ within the dark-fermion model in the heavy dark-sector scenario. The measured data being in agreement with the Standard Model prediction, this observation can be translated into 95\% CL exclusion limits on the production cross-sections and coupling parameters of the signal models. Figure 3 shows upper limits at $95 \% \mathrm{CL}$ on the cross section in the light and heavy darksector scenarios of both the dark-fermion and dark-Higgs models as a function of the mediator mass $m_{Z^{\prime}}$. The limits for the dark-fermion model assuming a light (heavy) dark-sector are in the range of $0.68-27 \mathrm{pb}(0.066-9.8 \mathrm{pb})$ while those for the dark-Higgs model are in the range of 0.80 $-5.5 \mathrm{pb}(0.064-2.4 \mathrm{pb})$. The corresponding upper limits on the coupling $g_{\mathrm{SM}}$ of the $Z^{\prime}$ mediator to Standard Model particles assuming that the $Z^{\prime}$ coupling to dark-sector particles is $g_{\mathrm{DM}}=1$ are shown in Figure 4. These are competetive with a direct search for $Z^{\prime} \rightarrow q \bar{q}$ decays [6].

\section{References}

[1] ATLAS Collaboration. Search for dark matter in events with a hadronically decaying vector boson and missing transverse momentum in pp collisions at $\sqrt{s}=13 \mathrm{TeV}$ with the ATLAS detector. arXiv:1807.11471, submitted to JHEP.

[2] ATLAS Collaboration. The ATLAS Experiment at the CERN Large Hadron Collider. JINST 3 (2008) S08003.

[3] L. Evans and P. Bryant. LHC Machine. JINST 3 (2008) S08001.

[4] M. Autran, K. Bauer, T. Lin, and D. Whiteson. Searches for dark matter in events with a resonance and missing transverse energy. Phys. Rev. D92 (2015) 035007.

[5] M. Cacciari, G. P. Salam, and G. Soyez. The anti- $k_{T}$ jet clustering algorithm. JHEP 04 (2008) 063.

[6] ATLAS Collaboration. Search for light resonances decaying to boosted quark pairs and produced in association with a photon or a jet in proton-proton collisions at $\sqrt{s}=13 \mathrm{TeV}$ with the ATLAS detector. arXiv:1801.08769, submitted to Phys. Lett. B. 

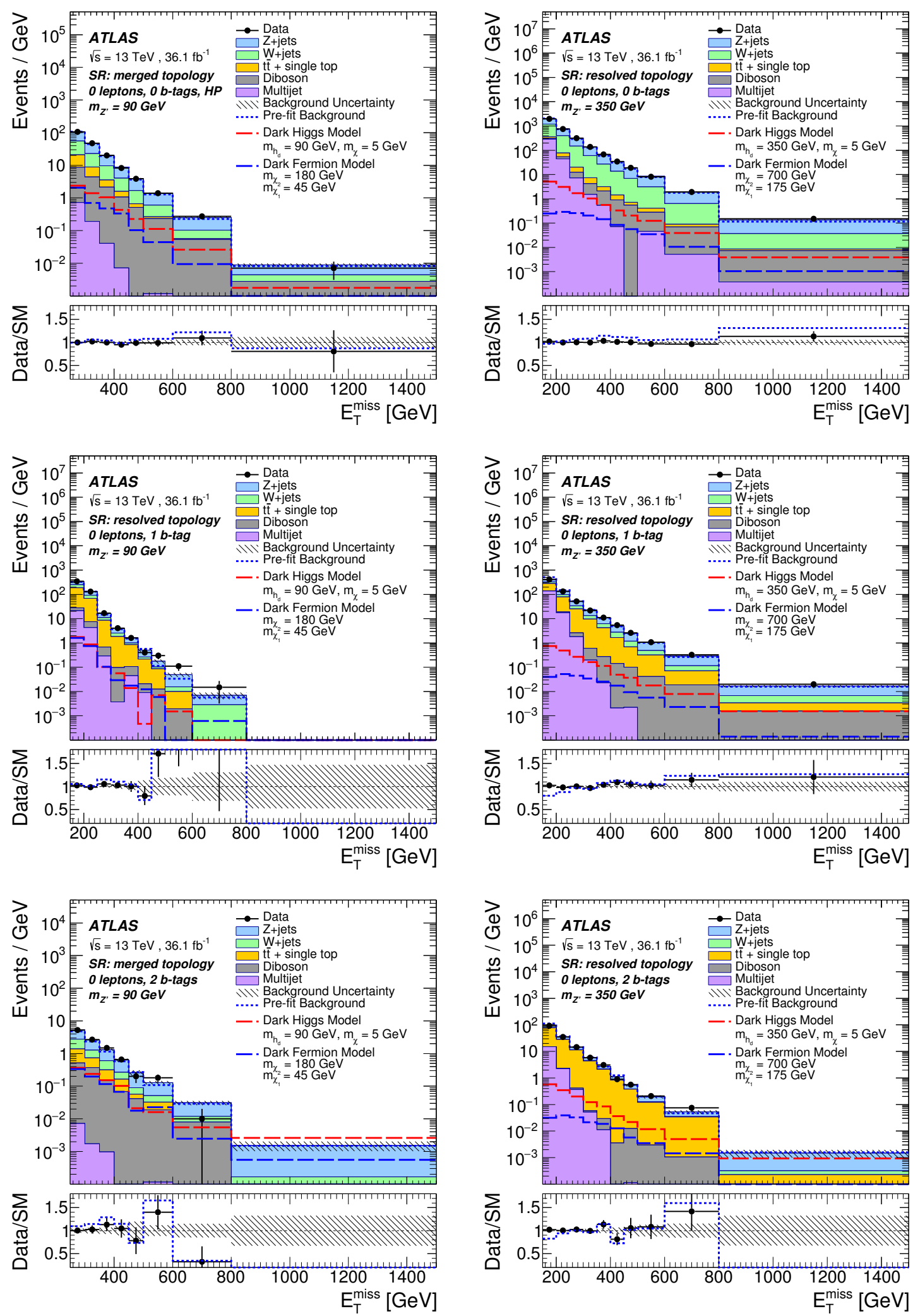

Figure 2: Selected distributions of $E_{\mathrm{T}}^{\mathrm{miss}}$ are shown for the event selection targeting the vector bosons with a mass of $90 \mathrm{GeV}$ (left) and $350 \mathrm{GeV}$ (right) in event categories with 0 (top), 1 (middle), and 2 (bottom) $b$-tagged jets in merged and resolved topologies [1]. 

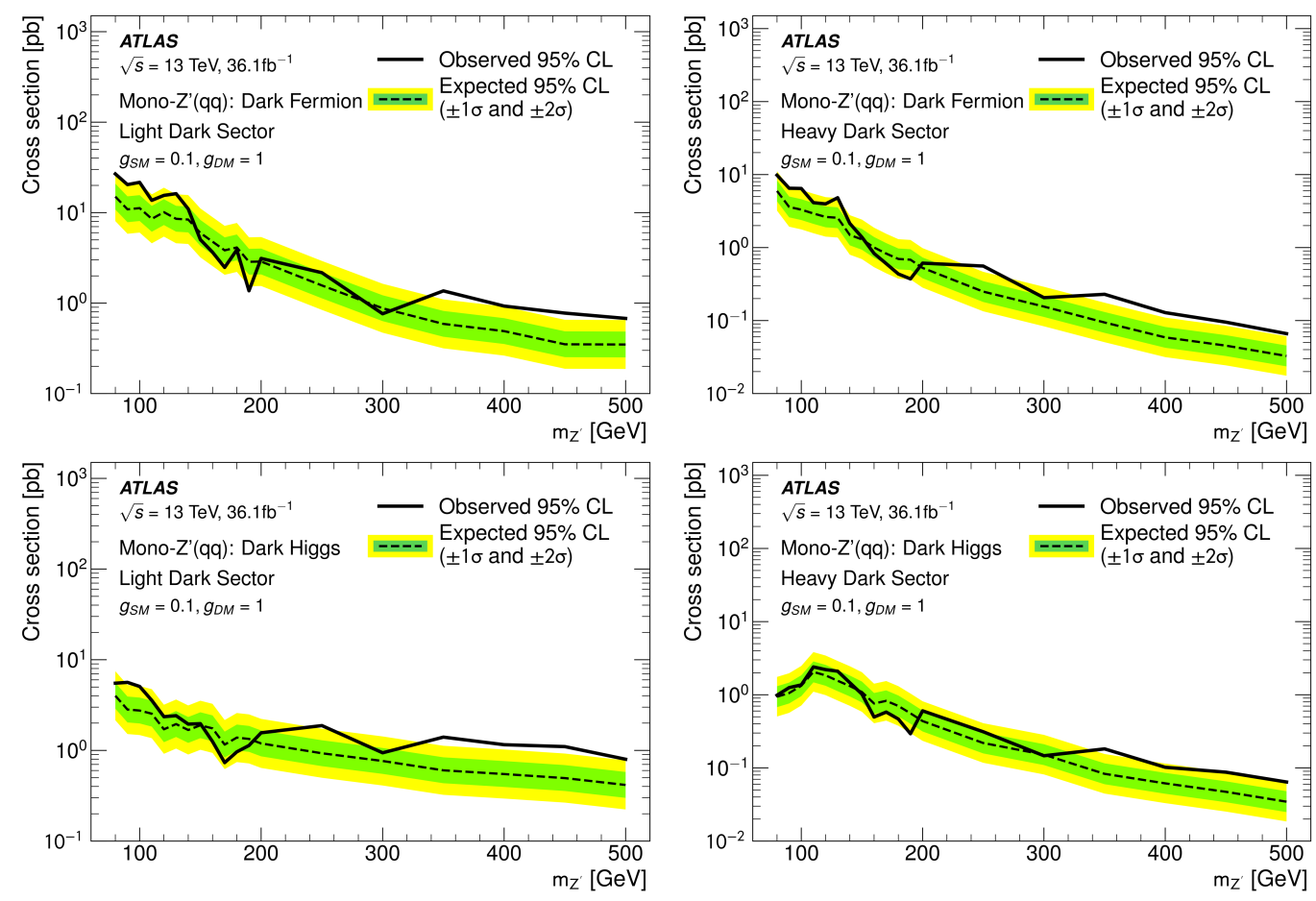

Figure 3: Upper limits at $95 \% \mathrm{CL}$ on the cross sections in the light (left) and heavy (right) dark-sector scenario of the dark-fermion (top row) and dark-Higgs (bottom row) models [1].
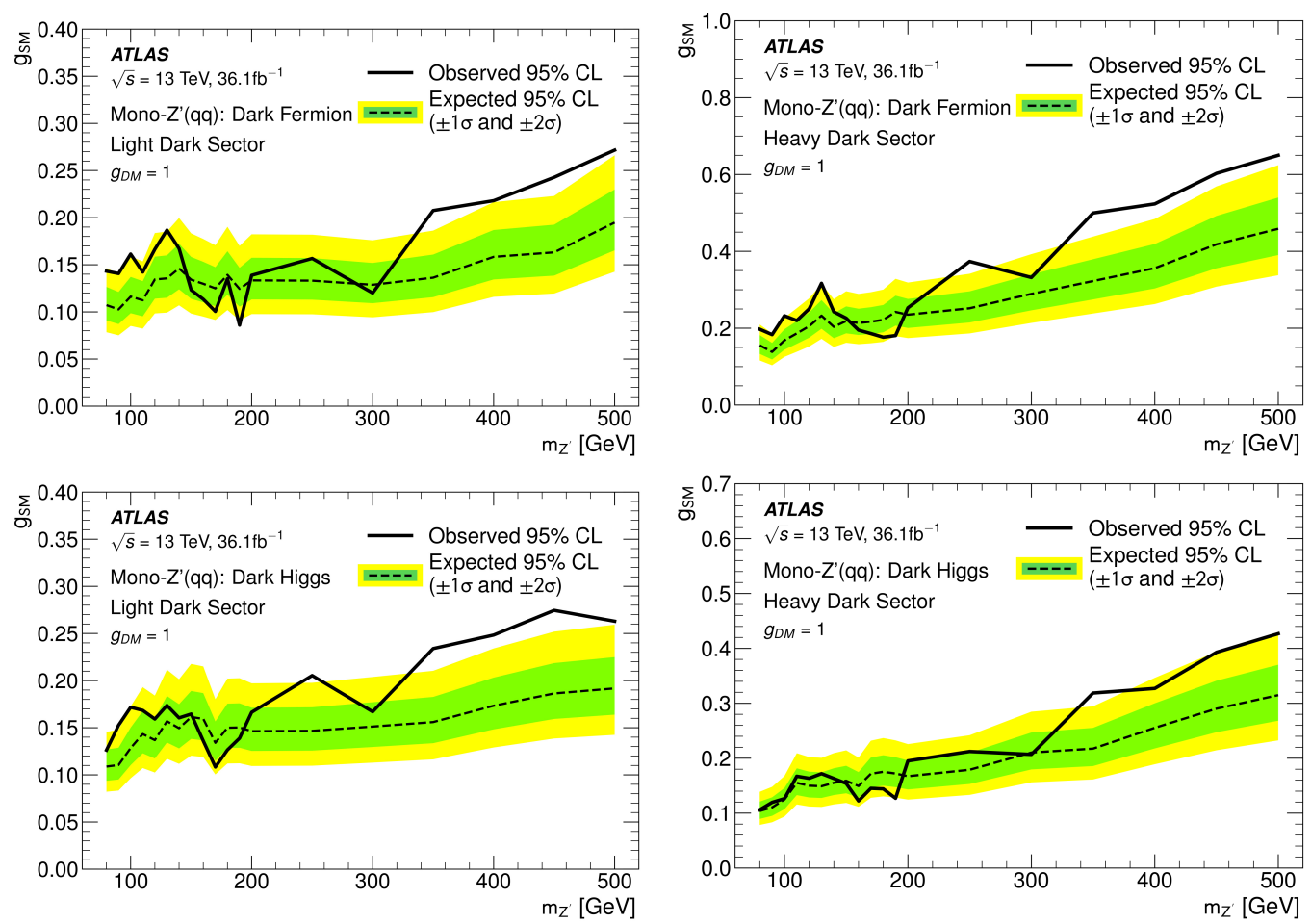

Figure 4: Upper limits at $95 \% \mathrm{CL}$ on the the product $g_{\mathrm{SM}} g_{\mathrm{DM}}$ of couplings $g_{\mathrm{SM}}$ of the mediator to the Standard Model and $g_{\mathrm{DM}}$ to dark-sector particles (the latter set to 1.0). They are shown for light (left) and heavy (right) dark-sector scenarios of the dark-fermion (top row) and dark-Higgs (bottom row) models [1]. 